\title{
Stochastic Ito-Calculus and Numerical Approximations for Asset Price Forecasting in the Nigerian Stock Market
}

\author{
Thomas Chinwe Urama1,2*, Patrick Oseloka Ezepue ${ }^{3}$ \\ ${ }^{1}$ Statistics, Information Modelling \& Financial Mathematics Research Group, Sheffield Hallam University, \\ Sheffield, UK \\ ${ }^{2}$ Department of Statistics, Institute of Management and Technology (IMT) Enugu, Enugu, Nigeria \\ ${ }^{3}$ Afriworld Higher Education \& Research Observatory 3E, Sheffield, UK \\ Email: ^Thomas.C.Urama@student.shu.ac.uk, *chinweurama@yahoo.com, patrick.ezepue@sky.com
}

How to cite this paper: Urama, T.C. and Ezepue, P.O. (2018) Stochastic Ito-Calculus and Numerical Approximations for Asset Price Forecasting in the Nigerian Stock Market. Journal of Mathematical Finance, 8, 640-667.

https://doi.org/10.4236/jmf.2018.84041

Received: September 27, 2018

Accepted: November 9, 2018

Published: November 12, 2018

Copyright $\odot 2018$ by authors and Scientific Research Publishing Inc. This work is licensed under the Creative Commons Attribution International License (CC BY 4.0).

http://creativecommons.org/licenses/by/4.0/

\begin{abstract}
Predicting prices of financial assets have always been topical in finance. This conceptual paper considers the seminal paper by Black-Scholes [1], how to determine the parameters of the geometric Brownian motion, and their use in forecasting stock prices, especially for cases where analytic solutions are not feasible. Generally describing stock market dynamics and heuristic modelling of derivative prices in the Nigerian Stock Market (NSM), the paper particularly uses data on the stock prices of a Nigerian bank to develop the stochastic calculus foundations of such modelling. The bank stock prices are part of daily closing stock prices of 82 stocks listed and fully traded in the NSM between 3 August 2009 and 26 August 2013, which support wider heuristic modelling foreshadowed by the paper. Technically, the paper considers the use of accurate numerical approximation method to simulate nonlinear solutions to stochastic differential Equations (SDE) resulting from asset prices. Importantly, the paper illustrates the workings of the standard Black-Scholes results as a preparation for more detailed empirical modelling of some candidate derivative pricing formulae in the Nigerian Stock Market (NSM). It particularly illustrates the dual use of the BS [1] model and the Euler-Maruyama (EM) model for pricing, respectively, the derivative and underlying assets in a financial market, for example the NSM. The paper will help the Nigerian Stock Exchange to use derivatives to deepen the NSM. The specific objectives of the paper and the notes on policy implications provide the rudiments of theory and follow-on heuristics for this goal. Also, academics and practitioners can use the results as starting points for enhancing the research and practice of derivative pricing in the NSM and other emerging markets, for
\end{abstract}


sectors and products of interest to them. The novelty of this line of work is that it has not been done so far in the NSM, and wider emerging African markets.

\section{Keywords}

Ito-Calculus, Brownian Motion, Volatility, Black-Scholes, Stochastic

Equations, Numerical Simulation, Convergence, Euler-Maruyama, Financial Assets Pricing

\section{Introduction}

Stochastic calculus deals with random motion of asset prices in financial engineering. It is useful in estimating the price of the underlying assets and in finding the equilibrium price of stock options [2]. This problem is not addressed satisfactorily in the Nigerian financial markets as far as derivative trading and asset pricing is concerned. This is because, unlike similar developing countries like Brazil, Malaysia, and Chile, including South Africa, derivatives trading has not been formally in place, having only been approved by the Central Bank of Nigeria $(\mathrm{CBN})$ in 2014. Consequently, the literature on derivative pricing and their stochastic calculus foundations by Nigerian and other authors are focused on other countries, as noted further below. Following the formal approval for derivatives trading in Nigeria, there is a need for research in key aspects of financial engineering that use data from Nigerian financial markets, for example the Nigerian Stock Market, and key sectors of the market, say, the banking sector. This paper fills this need, and is the first attempt known to the authors to do so since the said CBN approval.

The paper aims to explore relevant derivative pricing concepts that would enable a Nigerian bank to engage in derivatives trade. The specific objectives are to 1) determine the parameters of the geometric Brownian motion, and discuss their use in forecasting stock prices, especially for cases where analytic solutions are not feasible; 2) demonstrate the use of accurate numerical approximation methods to simulate nonlinear solutions to stochastic differential Equations (SDE) resulting from asset prices, using Ito stochastic and Stratonovich integrals; 3) illustrate the workings of the standard Black-Scholes results, as a preparation for more detailed empirical modelling of some candidate derivative pricing formulae in the Nigerian Stock Market (NSM) in subsequent work; and 4) particularly illustrate the dual use of the BS [1] model and the Euler-Maruyama (EM) model for pricing, respectively, the derivative and underlying assets in a financial market, for example the NSM. The backdrop to the aim and objectives is further explained below.

Theoretically, many stochastic processes are functionals of the Brownian motion process, for example the Black-Scholes [1] option pricing model. We explore the accuracies in approximating non-trivial analytical solutions to stochas- 
tic differential Equations (SDEs) numerically, with the Black-Scholes model as a focal point.

Recently in 2014 the Central Bank of Nigeria (CBN) approved derivatives trading in Nigerian capital markets, having earlier in 2012 provided a N40 million grant to the Nigerian Association of Securities Dealers (NASD) for developing an over-the-counter (OTC) derivatives trading platform. The approved pioneer products for take-off in the Nigerian Stock Market (NSM) include Foreign Exchange (FX) options, Forwards (Outright and Non-deliverables), FX swaps, and Cross-Currency interest rate swaps. Most of these products are OTC derivatives. Before this policy shift, derivatives such as FX forwards had been informally traded by Nigerian market participants, allowing dealers to do FX transactions among themselves and with retail/wholesale customers, [3]. In May 2014, the authors visited the Nigerian Stock Exchange for one-day collaborative discussions of the possible research development needs that would support wider systematic stock market characterisation and development (SSMCD) in the NSM, ([4] [5]). The NSE management identified derivatives research as one of the key areas of interest. They also indicated that the NSM is benchmarking its nascent derivatives market on the Johannesburg Stock Exchange (JSE), and confirmed the CBN support for the market.

The above-mentioned formalisation of derivatives trading aims to deepen the Nigerian capital markets and make the NSM more efficient. It will also enable market operators and end-users to hedge against losses arising from exchange rate fluctuations. Moreover, given the absence of formal derivatives trading in Nigeria, existing literature is focused on developed countries, and some developing where derivatives are traded, for example Brazil ([6] [7] [8]), Chile and Brazil [9]. This paper, therefore, examines aspects of the standard Black-Scholes derivative pricing model, and how it can be applied to the investment and risk-hedging interests of a Nigerian bank, referred to as Bank X.

[4] [5] note that six market features explored in empirical finance are germane for characterising the behaviour of a financial market, namely efficiency, volatility, bubbles, anomalies, valuations and predictability. The first four of these features are more meaningful to explore at the overall market level using the All Shares Index (ASI) as in these references. All the features are again important to study for key sectors of a market, and valuations and predictability are even more meaningful for individual firms, as part of their overall investment and risk management decisions. Such firm-specific analysis should use a firm's share prices and returns as in this paper. Consequently, for the banking sector, which is a primary sector of the NSM, some of these features are explored, [10]. The derivatives trading aspects are linked to asset price volatility, bank valuations, investments, and risk-hedging decisions, and are unexplored in the NSM, because of the lack of formal derivative trading mentioned above. This paper fills this gap by using data on a Nigerian bank's share prices to explore derivative pricing concepts. It is part of wider SSMCD work that could be said to pioneer formal derivatives research and applications in the NSM. 
The key theoretical contributions of the paper to knowledge include the explication of essential Ito and Stratonovich calculus underpinnings of derivative pricing, related understanding of the workings of the Black-Scholes [1] model, and relevant numerical methods approximations to derivative pricing models with non-analytic solutions. The practical contribution is mainly a demonstration of how these concepts inform the estimation of derivative and underlying asset prices of Bank $\mathrm{X}$ in Nigeria, and the implications of the results for future work aimed at enhancing derivative trading in Nigeria, to deepen the NSM.

The outline of the paper is as follows. Section 2 summarises relevant literature on stochastic calculus and its use in deriving underlying asset prices. Section 3 presents the methodology for estimating stock prices using historical volatility, and simulating an SDE Brownian motion using Euler-Maruyama method. Section 4 states and discusses the main results, especially numerical approximation for non-trivial SDE problems for which exact or analytical methods may not be applicable. The section adoptsa numerical related to Langevin equation whose solution is the Ornstein Uhlenbeck process, and briefly compares approximation errors in Euler-Maruyama and Milstein's approximations. Section 5 concludes the paper.

\section{Literature Review}

\subsection{Background Notes on Stochastic Calculus and Differential Equations}

Ito stochastic and Stratonovich integrals offer good numerical approximations to solutions of stochastic differential Equations (SDE) obtained from stock prices. They can also be used in estimating numerical solutions of other asset prices that serve as underlying stocks of derivative products, where analytic solutions are not feasible, and the price dynamics of these underlying assets are needed in the pricing of call and put options of the derivative assets, [11]. One of the methods for evaluating of these integral equations is the use of Euler-Maruyama approximation to solutions of differential and integral equations.

Whilst the BS [1] model and its extensions are ubiquitously applied to pricing derivative assets in other global markets, US, UK, Europe, Brazil, and South Africa, for example, such assets are yet to be fully introduced in Nigeria. Therefore, this paper is a first step in addressing an original problem of how to price non-existing derivative products, albeit heuristically. Follow-on work to this paper will approach the problem by benchmarking the Nigerian Stock Market (NSM) on the Johannesburg Stock Exchange (JSE), and using (for a simplified statement of the problem) comparative correlations of asset prices in the two markets to judge the degree of closeness of the markets and/or their sectors. This judgement will inform simulations of derivative prices that will fit the stylised facts of the NSM, [12]. These computational modelling under assumed scenarios requires an intimate understanding of closed-form numerical forecasting of asset prices which this paper explores. 
[13] assert that stochastic calculus is useful in many financial assets including derivatives and that they are used in solving Black-Scholes option pricing problems, market risk adjustment, and project valuation using method of [14]. Some numerical schemes for approximating SDEs are discussed in, ([15]-[20]). [21] note that what is common among them is the use of Ito-Taylor series expansion and Wiener integrals.

Ozaki ([22] [23]), however, point out some shortcomings associated with the numerical solutions of SDEs and other differential equations, for example lack of exact solutions to linear SDEs and inability to preserve qualitative features of the exact solution. [24] explores trivial/nontrivial and analytic/numerical order-ofconvergence solutions to SDEs. Hence, the key question for solving the NSMJSE derivative pricing problems noted above is: Under what conditions and for which numerical approximation schemes are these limitations overcome? This will facilitate suitable experimental modelling of potentially useful NSM derivatives.

[25] derives the Euler-Maruyama and Milstein method for numerical approximation to SDEs, with a MATLAB code for their implementation. [26] discusses the MATLAB algorithm to numerical simulations in a stochastic differential equation, while [11] use Ito and Stratonovich integrals in deriving security prices. [27] offers a major contribution in the computational solution of SDEs with special focus on strong and weak convergence of SDE solvers. We consider here a model representing the underlying asset prices in the form of an Ito SDE that takes the form

$$
\mathrm{d} S_{t}=\mu\left(S_{t}, t\right) \mathrm{d} t+\sigma\left(S_{t}, t\right) \mathrm{d} W_{t}, S(o)=S_{o}
$$

$\mu, \sigma>0, W_{t}$ is a Brownian motion.

Recall below the Black-Scholes [1] parabolic partial differential equation which is a stochastic calculus model for option price as a function of the underlying stock price at time $\mathrm{t}$

$$
\frac{\partial C}{\partial t}+\frac{1}{2} \sigma^{2} S^{2} \frac{\partial^{2} C}{\partial S^{2}}+r S \frac{\partial C}{\partial S}-r C=0,
$$

where $C(S, \tau, T)$ is the option price at time $\tau, r$ is the risk-free interest rate, $S$ is the underlying stock price, and $\sigma$ is the volatility of the stock price per annum.

\subsection{Theoretical Framework}

We study the analytic solution to the SDE (1) above, its associated numerical approximation, and its use in European option pricing. For a European option, which coincidently is the derivative option pricing formula proposed to be adopted for use in Nigerian Stock Market, Equation (2) is solved under the finite boundary condition that

$$
C(0, \tau)=0, C(S, T, T)=\operatorname{Max}\{S-K, 0\}
$$

and

$$
P(S, T, T)=\operatorname{Max}\{K-S, 0\},
$$


for call and put options, respectively, where $T$ is expiration date (maturity) of the option, and $K$ is its exercise (strike) price. Thus, the solution $C(S, \tau, T)$ to Equations (2) and (3) above is given by the call option value derived from [1] and [28] and written as

$$
\begin{aligned}
C(S, 0, T)= & S N\left\{\frac{\ln \left(\frac{S}{K}\right)+\left[r+\left(\frac{\sigma^{2}}{2}\right)\right](T-\tau)}{\sigma \sqrt{T-\tau}}\right\} \\
& -K \mathrm{e}^{-r(T-t)} N\left\{\frac{\ln \left(\frac{S}{K}\right)+\left[r-\left(\frac{\sigma^{2}}{2}\right)\right](T-\tau)}{\sigma \sqrt{T-\tau}}\right\}
\end{aligned}
$$

and $N(x)$ is the cumulative standard normal distribution given by

$$
N(x)=\frac{1}{\sqrt{2 \pi}} \int_{-\infty}^{x} \mathrm{e}^{-\frac{t^{2}}{2}} \mathrm{~d} t .
$$

The BS model is attractive in pricing applications because all the model parameters, except the volatility, are observable because the risk-free neutrality assumption in option theory allows us to substitute the risk-free interest rate, $r$, for the expected rate of return $\mu$, [29]. Estimating this unobservable volatility parameter usually constitutes the major problem in pricing financial derivatives. The Black-Scholes Equation (2) uses the geometric Brownian motion as the governing relation for the underlying asset stock price. It is based on the assumption that the stock price $S$ as a function of time (i.e. $S(t), t \in R^{+}$) satisfies the SDE (1), with $S(t)$ as the underlying stock price at time $t, \mu \in R$ is the rate of return (or drift), $\sigma \in R_{+}$is the volatility or variance of the stock(which is assumed to be constant throughout the duration of the option contract), $\mathrm{d} W(t)$ is the white noise/stochastic differential of a normalized Wiener process, and $\mathrm{d} S(t)$ is the change in security price over time. Observe that on re-arranging Equation (1) we shall obtain

$$
\frac{\mathrm{d} S}{S}=\mu \mathrm{d} t+\sigma \mathrm{d} W(t)
$$

where, $\frac{d S_{t}}{S_{t}}$ is the stock price return during the time $t$, under consideration.

\section{Ito formula in Brownian motion: Theorem 1}

Let $X(t)$ be an Ito process represented by the SDE

$$
\mathrm{d} X(t)=\alpha(t, X(t)) \mathrm{d} t+\beta(t, X(t)) \mathrm{d} W(t)
$$

Let $g(t, x)$ be twice differentiable function defined on $[0, \infty) \times \mathbb{R}$, or $g(t, x) \in C^{2}[0, \infty) \times \mathbb{R}$, then

$$
Y(t)=g(t, X(t))
$$

is also an Ito process and 


$$
\mathrm{d} Y(t)=\frac{\partial g}{\partial t}(t, X(t)) \mathrm{d} t+\frac{\partial g}{\partial x}(t, x(t)) \mathrm{d} X(t)+\frac{1}{2} \frac{\partial^{2} g(t, X(t))}{\partial x^{2}}[d X(t)]^{2}
$$

(proof omitted being common knowledge; see for example, [30]; more rigorously [31]: pp. 92-94; [32]: pp. 88-89).

Now relating the above second-order differential equation on $Y(t)$ to stock price and for any given function $g(t, x)$ as above, setting it equivalent to $f(S, t)=\ln S_{t}$, we have the general Ito formula

$$
\begin{aligned}
\mathrm{d} f(S, t) & =f_{s} \mathrm{~d} S+f_{t} \mathrm{~d} t+\frac{1}{2}\left\{f_{s s}(\mathrm{~d} S)^{2}+2 f_{s t} \mathrm{~d} S \mathrm{~d} t+f_{t t}(\mathrm{~d} t)^{2}\right\} \\
& =\frac{\partial f}{\partial S} \mathrm{~d} S+\frac{\partial f}{\partial t} \mathrm{~d} t+\frac{1}{2}\left\{\frac{\partial^{2} f}{\partial S^{2}}(\mathrm{~d} S)^{2}+2 \frac{\partial^{2} f}{\partial S \partial t} \mathrm{~d} S \mathrm{~d} t+\frac{\partial^{2} f}{\partial t^{2}}(\partial t)^{2}\right\}
\end{aligned}
$$

With $f=\ln S_{t}$ in the Brownian motion, $\mathrm{d} S \equiv \mathrm{d} S_{t}=\mu S(t) \mathrm{d} t+\sigma S(t) \mathrm{d} W_{t}$ we obtain from Equation (7),

$$
\begin{aligned}
& \mathrm{d} f(S, t)=\mathrm{d}\left(\ln S_{t}\right) \\
& =\frac{1}{S_{t}}\left(\mu S_{t} \mathrm{~d} t+\sigma S_{t} \mathrm{~d} W_{t}\right)+0 \cdot \mathrm{d} t \\
& \quad+\frac{1}{2}\left\{\frac{1}{-S_{t}^{2}}\left(\mu S_{t} \mathrm{~d} t+\sigma S_{t} \mathrm{~d} W_{t}\right)^{2}+2(0)\left(\mu S_{t} \mathrm{~d} t+\sigma S_{t} \mathrm{~d} W_{t}\right)+0(0)\right\}
\end{aligned}
$$

since, $\mathrm{d} t \mathrm{~d} t=\mathrm{d} t \mathrm{~d} W_{t}=\mathrm{d} W_{t} \mathrm{~d} t=0$

$$
\begin{aligned}
& =\mu \mathrm{d} t+\sigma \mathrm{d} W_{t}+\frac{1}{2}\left\{\frac{1}{-S_{t}^{2}}\left(\sigma^{2} S_{t}^{2} \mathrm{~d} t\right)\right\} \\
& =\mu \mathrm{d} t+\sigma \mathrm{d} W_{t}-\frac{\sigma^{2}}{2} \mathrm{~d} t \\
& =\left(\mu-\frac{\sigma^{2}}{2}\right) \mathrm{d} t+\sigma \mathrm{d} W_{t}
\end{aligned}
$$

But $\left[\ln S_{t}\right]=\frac{1}{S_{t}} \mathrm{~d} S_{t}=\frac{\mathrm{d} S_{t}}{S_{t}}$ or the stock price return as in Equation (6), hence on taking the stochastic integral of the expression

$$
\begin{gathered}
\mathrm{d}\left[\ln S_{t}\right]=\frac{\mathrm{d} S_{t}}{S_{t}}=\left[\mu-\frac{\sigma^{2}}{2}\right] \mathrm{d} t+\sigma \mathrm{d} W_{t} \\
\Rightarrow \ln \left(S_{t}\right)-\ln S_{t}=\int_{0}^{t}\left[\mu-\frac{\sigma^{2}}{2}\right] \mathrm{d} t+\int_{0}^{t} \sigma \mathrm{d} W_{t}
\end{gathered}
$$

or

$$
\begin{gathered}
\ln \left(\frac{S_{t}}{S_{0}}\right)=\left(\mu-\frac{\sigma^{2}}{2}\right) t+\sigma W_{t} \\
\Rightarrow \frac{S_{t}}{S_{0}}=\exp \left\{\left[\mu-\frac{\sigma^{2}}{2}\right] t+\sigma W_{t}\right\} \text { or } S_{t}=S_{0} \exp \left\{\left[\mu-\frac{\sigma^{2}}{2}\right] t+\sigma W_{t}\right\}
\end{gathered}
$$

Lemma 1:

In any plain vanilla option, the evolution of a firm's stock price for the under- 
lying stock is given by a geometric Brownian motion

$$
\mathrm{d} S_{t}=\mu\left(S_{t}, t\right) \mathrm{d} t+\sigma\left(S_{t}, t\right) \mathrm{d} W_{t}, S(o)=S_{o},
$$

if and only if the exact solution of the Brownian motion is given by

$$
S_{t}=S_{0} \exp \left\{\left[\mu-\frac{\sigma^{2}}{2}\right] t+\sigma W_{t}\right\}
$$

where, $\mu, \sigma, W_{t}$ denote respectively drift of the asset return, its volatility and the Wieners process which is the random perturbation affecting the evolution of the process.

Proof: By [1] seminal paper, the underlying stock price for derivative option pricing follows a geometric Brownian, is log normally distributed, and could be represented by Equation (2) and satisfies a certain second order differential equation represented by Equation (7).

For this we set $F(S, t)=\log S$, and by Ito's lemma and Taylor series,

$$
\begin{aligned}
& \mathrm{d} F(S, t)=\mathrm{d}(\log S) \\
& =\frac{\partial F}{\partial S} \mathrm{~d} S+\frac{\partial F}{\partial t} \mathrm{~d} t+\frac{1}{2} \frac{\partial^{2} F}{\partial S^{2}}(\mathrm{~d} S)^{2}+\frac{\partial^{2} F}{\partial S \partial t} \mathrm{~d} S \mathrm{~d} t+\frac{1}{2} \frac{\partial^{2} F}{\partial t^{2}}(\mathrm{~d} t)^{2}
\end{aligned}
$$

(since all other higher powers of the derivatives vanish by Ito formula after the second order)

$$
\begin{aligned}
\mathrm{d}(\log S)= & \frac{\partial F}{\partial S}(\mu S \mathrm{~d} t+\sigma S \mathrm{~d} W)+\frac{\partial F}{\partial t} \mathrm{~d} t+\frac{1}{2} \frac{\partial^{2} F}{\partial S^{2}}(\mu S \mathrm{~d} t+\sigma S \mathrm{~d} W)^{2} \\
& +\frac{\partial^{2} F}{\partial S \partial t} \mathrm{~d} S \mathrm{~d} t+\frac{1}{2} \frac{\partial^{2} F}{\partial t^{2}}(\mathrm{~d} t)^{2}
\end{aligned}
$$

But $\frac{\partial F}{\partial S}=\frac{1}{S}, \frac{\partial^{2} F}{\partial S^{2}}=-\frac{1}{S^{2}}, \frac{\partial F}{\partial t}=0 \quad$ and $\quad\left(\mathrm{d} t^{2}\right)=\mathrm{d} t \mathrm{~d} t=0, \quad \mathrm{~d} t \mathrm{~d} W=0=\mathrm{d} W \mathrm{~d} t ，$ $\mathrm{d} W^{2}=\mathrm{d} W \mathrm{~d} W=\mathrm{d} t$; so that

$$
\mathrm{d}(\log S)=\left(\mu-\frac{\sigma^{2}}{2}\right) \mathrm{d} t+\sigma \mathrm{d} W
$$

Integrating both sides with respect to $t, t \in[0, T]$ yield

$$
\begin{aligned}
& \log S(t)-\log S(0)=\left(\mu-\frac{\sigma^{2}}{2}\right) t+\sigma W_{t} \\
& \Rightarrow S_{t}=S_{0} \exp \left\{\left[\mu-\frac{\sigma^{2}}{2}\right] t+\sigma W_{t}\right\}
\end{aligned}
$$

For the converse, suppose that the solution of the geometric Brownian motion is given by

$$
S_{t}=S_{0} \exp \left\{\left[\mu-\frac{\sigma^{2}}{2}\right] t+\sigma W_{t}\right\} \equiv S_{t}=S_{0} \exp \left\{\left[\mu-\frac{\sigma^{2}}{2}\right] t+\sigma \int_{0}^{t} \mathrm{~d} W\right\}
$$

(since $W(0)=0$ in a Brownian motion)

We will use Ito's lemma to establish that $S(t)$ as above satisfies Equation (L.1). For this purpose, we set 


$$
\begin{gathered}
F(t, W)=S_{0} \exp \left[\left(\mu-\frac{\sigma^{2}}{2}\right) t+\sigma \int_{0}^{t} \mathrm{~d} W\right] \\
\frac{\partial F}{\partial t}=S_{0} \exp \left[\left(\mu-\frac{\sigma^{2}}{2}\right) t+\sigma \int_{0}^{t} \mathrm{~d} W\right]\left(\mu-\frac{\sigma^{2}}{2}\right)=S(t)\left(\mu-\frac{\sigma^{2}}{2}\right) \\
\frac{\partial F}{\partial W}=S_{0} \exp \left[\left(\mu-\frac{\sigma^{2}}{2}\right) t+\sigma \int_{0}^{t} \mathrm{~d} W\right](\sigma)=\sigma S(t) \\
\frac{\partial^{2} F}{\partial W^{2}}=S_{0} \exp \left[\left(\mu-\frac{\sigma^{2}}{2}\right) t+\sigma \int_{0}^{t} \mathrm{~d} W\right]\left(\sigma^{2}\right)=\left(\sigma^{2}\right) S(t)
\end{gathered}
$$

Invoking the Ito formula and Equations (E.1) - (E.4), we have

$$
\begin{gathered}
\mathrm{d} S=\mathrm{d} F=\frac{\partial F}{\partial t} \mathrm{~d} t+\frac{\partial F}{\partial W} \mathrm{~d} W+\frac{1}{2} \frac{\partial^{2} F}{\partial W^{2}} \mathrm{~d} W^{2} \\
=S(t)\left(\mu-\frac{\sigma^{2}}{2}\right) \mathrm{d} t+\sigma S(t) \mathrm{d} W+\frac{\sigma^{2}}{2} S(t)(\mathrm{d} W)^{2} \\
=\mu S(t) \mathrm{d} t-S(t) \frac{\sigma^{2}}{2} \mathrm{~d} t+\sigma S(t) \mathrm{d} W+\frac{\sigma^{2}}{2} S(t) \mathrm{d} t \\
=\mu S(t)+\sigma S(t) \mathrm{d} W \\
\Rightarrow \mathrm{d} S=\mu S(t) \mathrm{d} t+\sigma S(t) \mathrm{d} W \text { as required } \\
\text { In general Equation }(\mathrm{L} .1) \text { can be written as } \\
\Delta f=\mathrm{d} f(s, t)=\frac{\partial f}{\partial S} \Delta S+\frac{\partial f}{\partial t} \Delta t+\frac{\partial f}{\partial t} \Delta \sigma_{t}+\frac{\partial f}{\partial t} \Delta r_{t}+\frac{1}{2} \frac{\partial^{2} f}{\partial S^{2}}\left(\Delta S_{t}\right)^{2}+\cdots
\end{gathered}
$$

and this last equation represents the various risks that an option is exposed to. The terms on the right-hand side represent: for the first term, the risk associated with the underlying stock, delta given by $\Delta S$, that of the change in time, theta or $\Delta t$, volatility change vega given by $\Delta \sigma$, interest rate rho is represented by $\Delta r$, and finally is gamma the second derivative of delta represented by $\left(\Delta S_{t}\right)^{2}$.

\section{Methodology}

SDEs are standard models for diffusion processes in physical and biological sciences, economics and finance, [27]. This paper uses them in form of Euler-Maruyama approximations of drift and volatility parameters, $\mu$ and $\sigma$ (which is a measure of risk) associated with future stock prices, based on the Black-Scholes formula and other models for option pricing. For example, the Langevian equation or Ornstein Uhlenbeck process are SDEs whose diffusion coefficients represent price volatility. The numerical solution to the price returns of Bank X asset was used to illustrate these procedures in the NSM. Hence, we can predict the future trajectory of any given asset price by this technique, which is important for developing derivatives trading in Nigerian financial markets.

\subsection{Estimating Stock Price Using Historical Volatility}

Given historical stock price data at some fixed intervals, for example days, 
weeks, or months, we can estimate the volatility or standard deviation of stock returns for use in the Black-Scholes option pricing formula. The values of drift and volatility so obtained can be used to evaluate analytical solutions and numerical approximations to SDE of the underlying stock price in a European call option. In the Black-Scholes model it is assumed that volatility of the underlying stock is constant and one of the ways of estimating this parameter is through historical volatility. In this section, we estimate the solutions of SDEs analytically by first computing the diffusion coefficient/historical volatility of the underlying stock return. For stock price data obtained form $(n+1)$ periods (usually in days), the estimate of historical volatility is given by

$$
\hat{\sigma}=\frac{\sqrt{\frac{1}{n-1} \sum_{i=1}^{n}\left(u_{i}-\bar{u}\right)^{2}}}{\sqrt{\tau}} \text {, where } u_{i}=\ln \left(\frac{S_{i}}{S_{i-1}}\right)
$$

$\bar{u}$ is the sample average $u_{i}, S_{i}$ is the stock price period $I$ and $\tau$ is the total length of each period in years. The annualized estimate of this standard deviation could also be written as

$$
\hat{\sigma}=\frac{S}{\sqrt{\tau}}
$$

where, $S=\sqrt{\frac{1}{n-1} \sum_{i=1}^{n} u_{i}^{2}-\frac{1}{n(n-1)}\left(\sum_{i=1}^{n} u_{i}\right)^{2}}$.

\section{Remark:}

We adopt the daily prices of Bank X asset in the NSM for this trial estimation.

In discrete time, the rentability of stock $S(t)$ or the stock price return over an interval $\left(t_{i-1}, t_{i}\right)$ is $R\left(t_{i}\right)=\frac{S\left(t_{i}\right)-S\left(t_{i-1}\right)}{S\left(t_{i-1}\right)}, i \geq 1$, and in continuous time the stock price return at any given time $t$ is

$$
R(t)=\frac{\mathrm{d} S}{S}=\mu \mathrm{d} t+\sigma \mathrm{d} W(t) .
$$

Evans (2003) asserts that given a differential equation:

$$
\frac{\mathrm{d} S(t)}{\mathrm{d} t}=\alpha(S(t), t)+B(S(t), t) \varepsilon(t), S(0)=S_{0}
$$

with $S():.[0, \infty) \rightarrow \mathbb{R}^{n}$ random function,

$$
\alpha: \mathbb{R}^{n} \times[0, T] \rightarrow \mathbb{R}^{n}, \quad B: \mathbb{R}^{n} \times[0, T] \rightarrow \mathcal{M}_{n \times m}(\mathbb{R}), \varepsilon: \mathbb{R} \rightarrow \mathbb{R}^{m}
$$

m-dimensional white noise defines a stochastic differential equation:

$$
\mathrm{d} S(t)=\alpha(S(t), t) \mathrm{d} t+B(S(t), t) \mathrm{d} W(t), S(0)=S_{0},
$$

if the white noise is solution of an $\mathrm{m}$-dimensional Wieners process. The integral form of Equation (11b) is therefore given by:

$$
S(t)=S_{0}+\int_{0}^{t} \alpha(S(u), u) \mathrm{d} u+\int_{0}^{t} B(S(u), u) \mathrm{d} W(u), \forall t \geq 0
$$

The major problem, therefore, is how to calculate the third term in Equation 
(12) above. Hence, we use Monte-Carlo simulation in MATLAB to estimate the Brownian (Wiener)

Figure 1 is a representation of typical stock price using Brownian motion which shows the degree of price predictability for given asset price dynamics. It shows that in most cases the underlying stock price dynamics can only be predicted in the short run and that the degree of accuracy in the prediction diminishes as the period under consideration increases.

Process, as shown in 3.1 mfile below.

\subsection{Mfile1: Brownian Path Simulation}

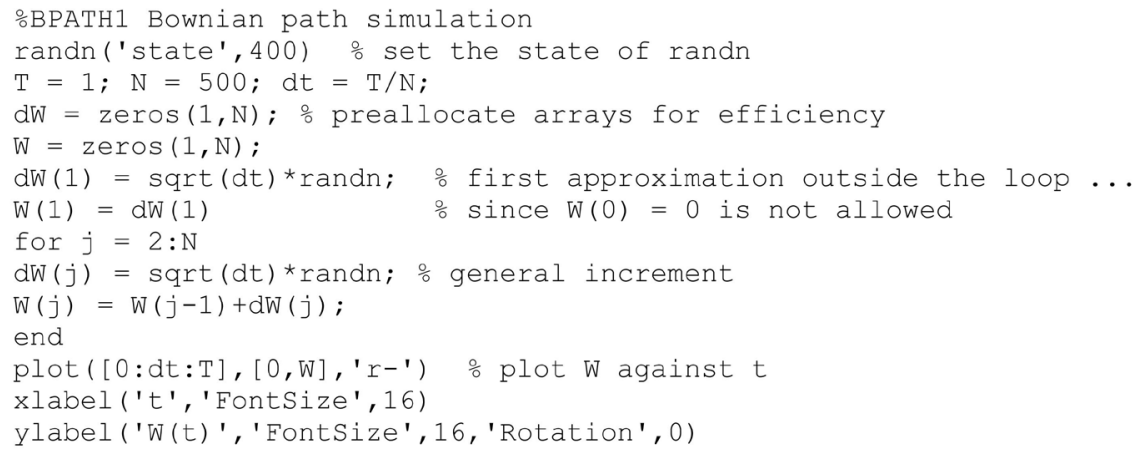

Figure 1 describes the stochastic nature of stock market prices in Nigeria and is typical of stock prices in most markets. It shows the Brownian motion character of process whereby the sample paths widely fluctuating and continuous.

\subsection{Using Euler-Maruyama Method to Estimate Stock Return}

We recall that a numerical solution to an ordinary differential equation can be obtained through Euler's method. Similarly, one can numerically obtain the solution to an SDE which is a continuous-time stochastic process by Euler-Maruyama

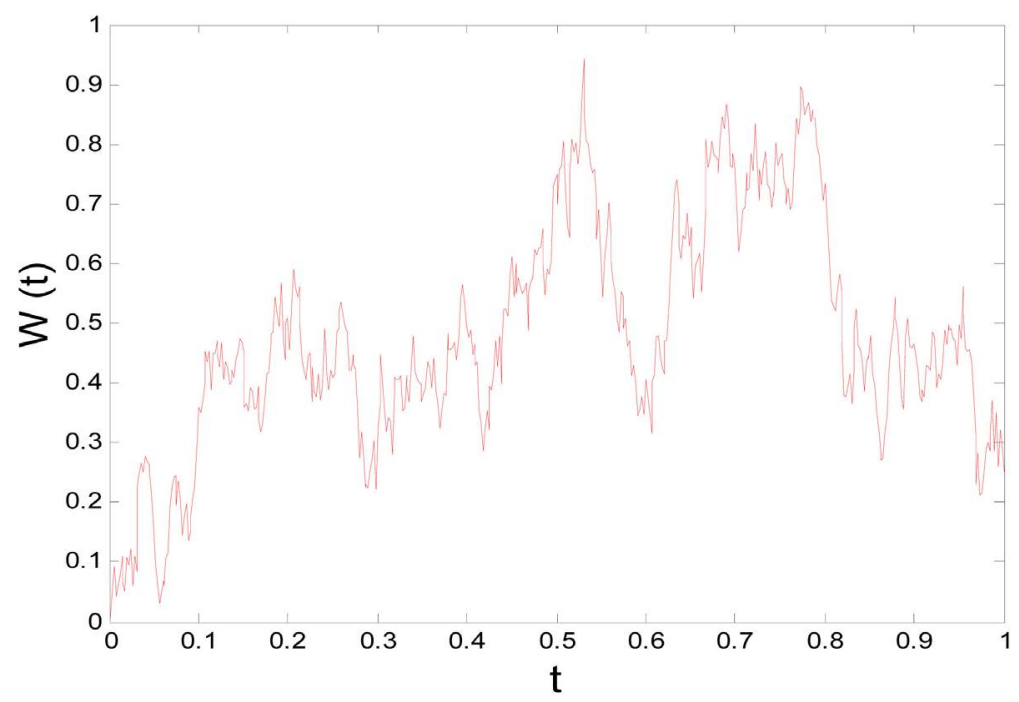

Figure 1. Brownian path simulation. 
(EM) or Milstein approximations of Black-Scholes related SDEs, since very few SDEs have closed form solutions, just like the ODEs. We usually solve nonlinear SDEs when no known analytical solution is available, by simulating the Wiener's stochastic process, [33] and [34]. Recognising that stochastic processes like the Brownian motion are continuous but non-differentiable, Dunbar states that with

$$
\mathrm{d} X(t)=f(X(t)) \mathrm{d} t+g(X(t)) \mathrm{d} W(t), X\left(t_{0}\right)=X_{0}
$$

with step size $\mathrm{d} t$, we can approximate and simulate the given Equation (13) with the relation

$$
X_{j}=f\left(X_{j-1}\right) \mathrm{d} t+g\left(X_{j-1}\right)\left[W\left(t_{j-1}+\mathrm{d} t\right)-W\left(t_{j-1}\right)\right]
$$

or equivalent to

$$
X_{j}=f\left(X_{j-1}\right) \mathrm{d} t+g\left(X_{j-1}\right)\left[W\left(t_{j}\right)-W\left(t_{j-1}\right)\right]
$$

\section{Results and Discussion}

\subsection{The Euler-Maruyama Method}

A given stochastic differential equation:

$$
\mathrm{d} X(t)=f(X(t)) \mathrm{d} t+g(X(t)) \mathrm{d} W(t), X(0)=X_{0}, 0 \leq t \leq T
$$

in Equation (13) above could be transformed into a stochastic integral equation written as

$$
X(t)=X_{0}+\int_{0}^{t} f(X(s)) \mathrm{d} s+\int_{0}^{t} g(X(s)) \mathrm{d} W(s)
$$

where, $f$ and $g$ are scalar functions, the initial condition $X_{0}$ is a random variable, and similarly the solution $X(t)$ is also a random variable for every $t$. If, however, $g \equiv 0$ and $X_{0}$ is a constant, then the problem reduces to a deterministic case which is an ordinary differential equation, and the solution will be Euler's approximation. Because of the stochastic component of the Equation (13) above, the Euler-Maruyama method makes use of Ito integral calculus. To apply numerical solution to the SDE, over any prescribed interval $[0, T]$, we discretize the interval. We set $\Delta t=\frac{T}{L}$ for some positive integer $L$, and $\tau_{j}=j \delta t$ for all $j=0,1,2, \cdots, L$; And suppose we denote $t=\tau_{j}$ and $t=\tau_{j-1}$ in Equation (14) we obtain:

$$
\begin{gathered}
X\left(\tau_{j}\right)=X_{0}+\int_{0}^{\tau_{j}} f(X(s)) \mathrm{d} s+\int_{0}^{\tau_{j}} g(X(s)) \mathrm{d} W(s) \text { for } t=\tau_{j} \\
X\left(\tau_{j-1}\right)=X_{0}+\int_{0}^{\tau_{j-1}} f(X(s)) \mathrm{d} s+\int_{0}^{\tau_{j-1}} g(X(s)) \mathrm{d} W(s) \text { for } t=\tau_{j-1}
\end{gathered}
$$

and on subtracting we obtain:

$$
X\left(\tau_{j}\right)=X\left(\tau_{j-1}\right)+\int_{\tau_{j-1}}^{\tau_{j}} f(X(s)) \mathrm{d} s+\int_{\tau_{j-1}}^{\tau_{j}} g(X(s)) \mathrm{d} W(s) \text { for } t=\tau_{j}
$$


by setting $X\left(\tau_{j}\right)=X_{j}$ we shall have:

$$
X_{j}=X_{j-1}+\delta t f\left(X_{j-1}\right)+g\left(X_{j-1}\right)\left[W\left(\tau_{j}\right)-W\left(\tau_{j-1}\right)\right]
$$

This SDE drives the stock price dynamics for a security asset in the NSM, say, with mean return $\mu=2$ and volatility (sigma) $=1$. We need an actual evolution of a firm's stock price prices to approximate the solution of Equation (6) above, after estimating the expectation and volatility parameters. For this, we use the Euler-Maruyama method to simulate the SDE by Monte-Carlo approach.

\subsection{Mfile 2: for Exact and Euler-Maruyama Approximation}

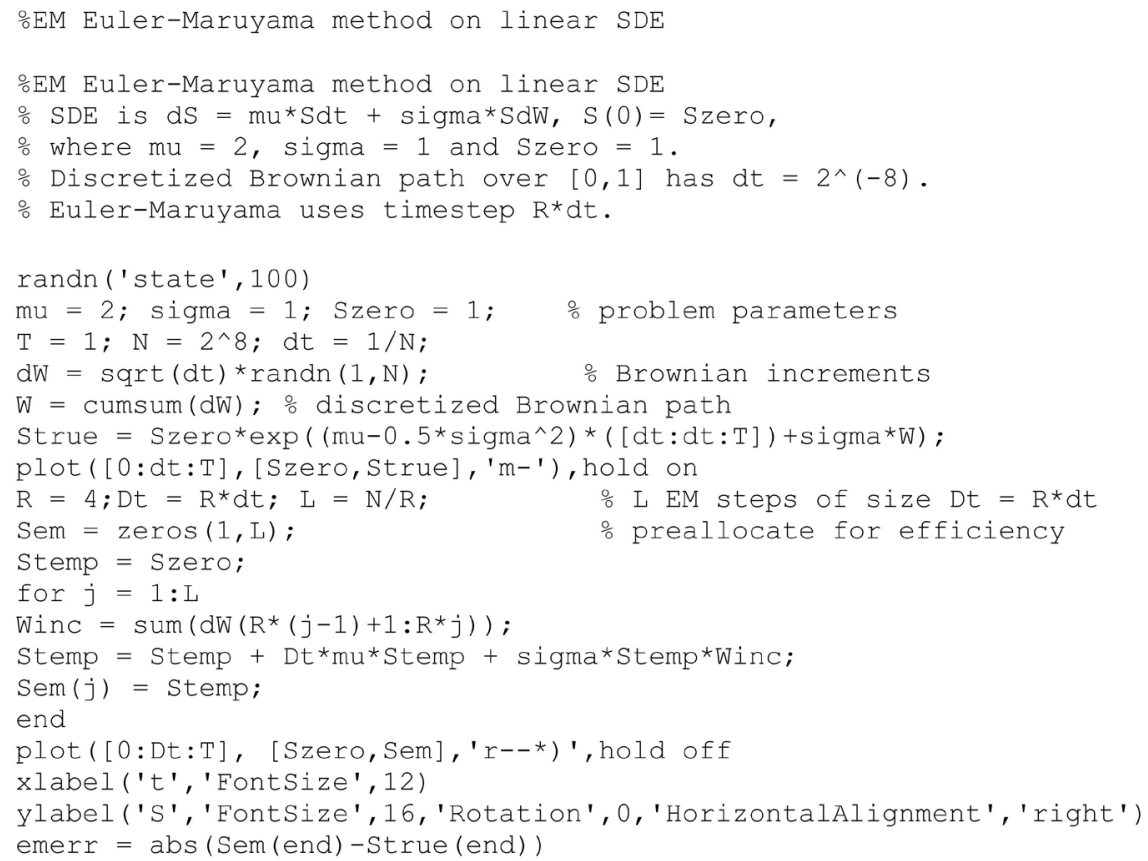

In Figure 2, the red star marks with dashed lines represent the Euler Maruyama approximations of the described stock prices in Figure 1, whereas the magenta line is the exact solution to the stock prices under consideration. It shows, therefore, that the approximations are reasonably good to enable the Black-Scholes derivative pricing scheme to apply in the Nigerian setting. The remaining figures maintain this choice of symbols for approximate and exact solutions but with different values of the parameter $\mathrm{R}$ which shows increasing accuracy as $\mathrm{R}$ decreases.

From Figure 2, the accuracy in using Equation (16) compared to the analytical solution represented by Equation (8) depends largely on the value of ' $R$ ' in the above MATLAB solutions. Greater accuracy is obtained for smaller values of " $R$ ". For instance, if we put $\mathrm{R}=2$ in the EMcode above, we approximate Equation (16) compared to the analytical solution as follows. As a practical illustration using Nigerian Bank X data from NSM, for instance (see appendix), we obtain the following mfile 3 below. Note that since the value of drift and variance are negligible, the estimated Euler-Maruyama and Exact solutions coincide. 


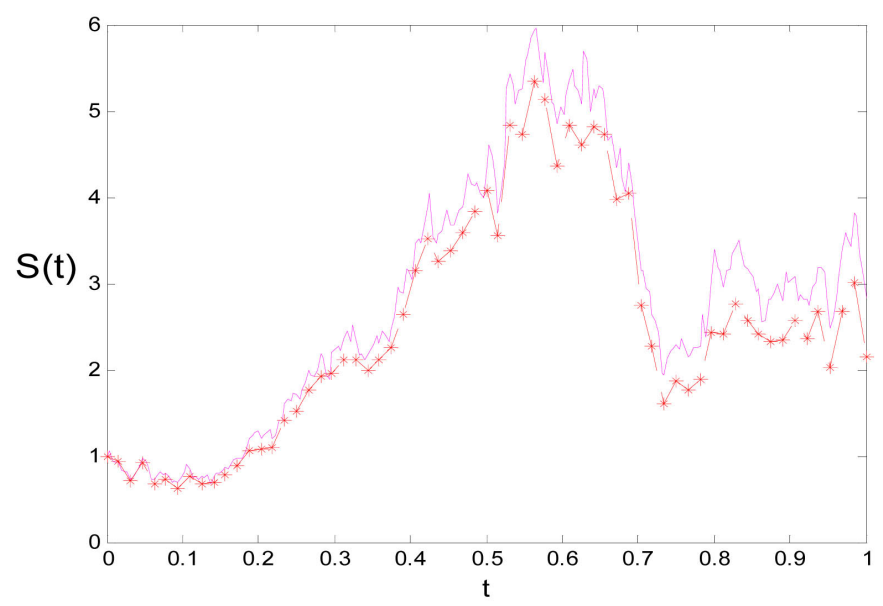

Figure 2. Exact and EM approximation.

Consider mean return and volatility data from a related study on Bank X financial asset, [35]. The mean parameters are as follows drift, $\mu=0.000599$, volatility, $\sigma=0.001$ (which are much smaller than 2 and 1, respectively), and Szero $=1.41$ given by using Equations (9) and (10).

These facts show that these approximation techniques are indicatively useful for derivatives modelling in the NSM. Related future work that benchmarks NSM derivatives on JSE price dynamics could use information on the crossmarket correlations of similar assets for which the derivatives prices are already known in the JSE to reverse-estimate plausible derivative prices in the NSM. This wider goal, not pursued further in this paper, requires further heuristic assumptions regarding the NSM-JSE correlational dynamics based on Random Matrix Theory (RMT) ([36] [37] [38]). Hence, for this ultimate research interest, these references provide the RMT results, which will be combined with ideas in this paper to implement the heuristics and price derivative price determinations involved.

\subsection{Mfile4: for Exact and Euler-Maruyama Approximation}

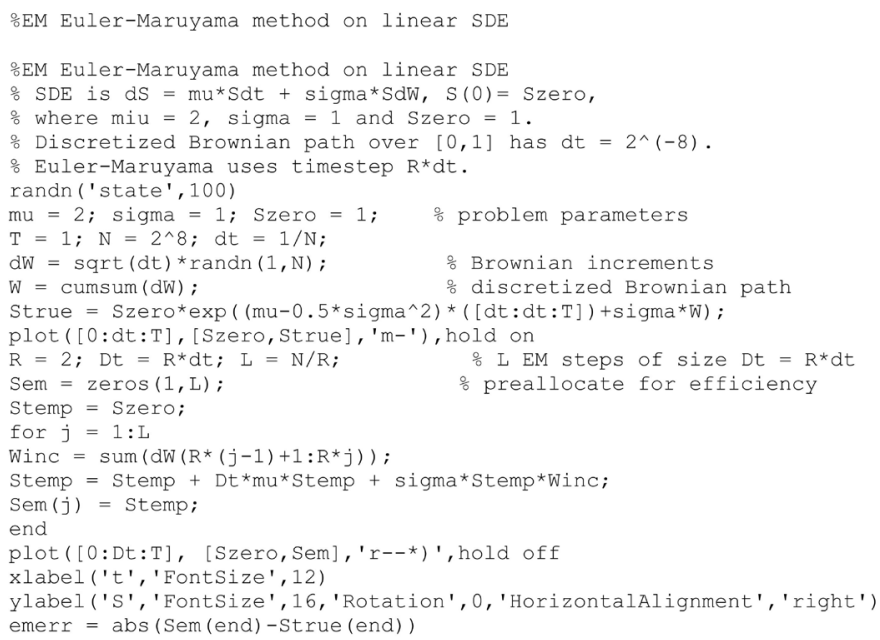


Figure 3 explores a choice of " $R$ " to obtain Euler-Maruyama approximation near enough to exact solution of the stochastic differential equation.

Using similar code as above for $\mathrm{R}=1$, we obtain an even better approximation thus.

Figure 4 illustrates further the earlier statement that smaller " $R$ " offers an approximate solution very close to the exact (analytical) solution of the stochastic differential equation. Thus, depending on the existence or otherwise of the analytical solution to a given differential equation of interest one can compare the two solutions and make the numerical approximation as close to the analytical solution depending on the choice(s) of the value(s) of " $R$ ". However, when the analytical solutions are not tenable one has to rely solely on the numerical approximation and the best choice or " $R$ " value should be mastered through practice.

The approximation accuracy of the EM method means that market makers and investors can use the model in predicting derivative asset prices, given primitive (Bank X) security as the underlying asset under which the contract (call or put option) is established. This could be carried out using, for instance, the Black-Scholes option pricing formula or its extensions. We now demonstrate the use of the above code in predicting the Bank $\mathrm{X}$ stock price using data obtained from Cashcraft database for NSM securities.

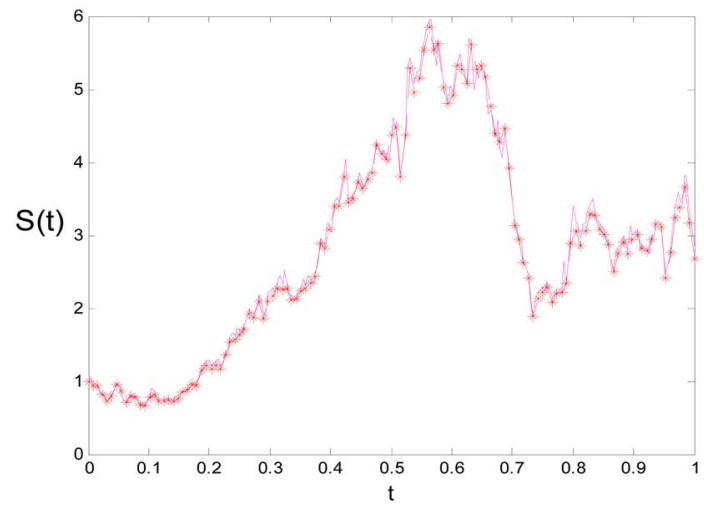

Figure 3. Exact and EM approximation for $\mathrm{R}=2$.

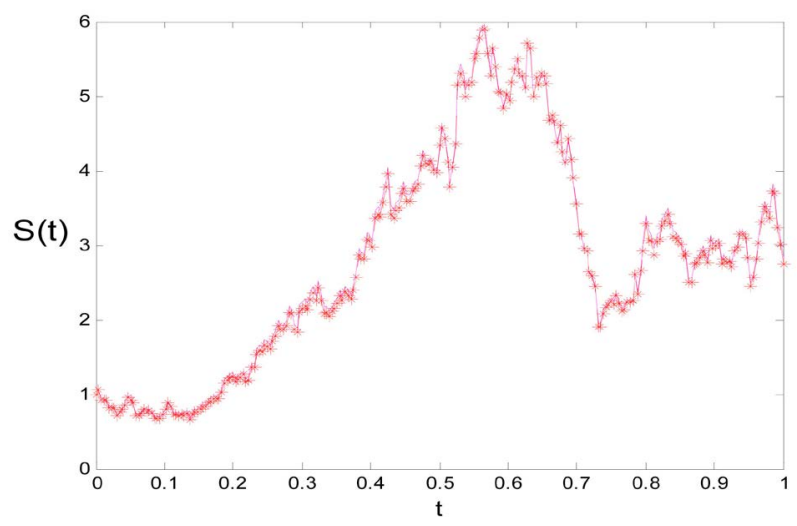

Figure 4. Coincident exact and EM approximation for $\mathrm{R}=1$. 


\subsection{Mfile3: Exact and Euler-Maruyama Approximations Using a} Sample of Bank X Data

\begin{tabular}{|c|c|c|c|c|c|}
\hline $\begin{array}{c}\text { Day of } \\
\text { the week }\end{array}$ & Date & $\begin{array}{l}\text { Price of } \\
\text { Stock, Si }\end{array}$ & $\begin{array}{c}\text { Price Rel. } \\
\mathrm{Si} / \mathrm{Si}-1\end{array}$ & $\begin{array}{l}\text { Daily Ret. } \\
\ln (\mathrm{Si} / \mathrm{Si}-1)\end{array}$ & $\begin{array}{c}\text { Daily Ret. } \\
\text { Squared }\end{array}$ \\
\hline Monday & $09 / 05 / 2016$ & 4.28 & & & \\
\hline Tuesday & $10 / 05 / 2016$ & 4.3 & 1.0046729 & 0.004662013 & $2.17344 \mathrm{E}-05$ \\
\hline Wed. & $11 / 05 / 2016$ & 4.31 & 1.00232558 & 0.002322881 & $5.39578 \mathrm{E}-06$ \\
\hline Thursday & $12 / 05 / 2016$ & 4.46 & 1.03480278 & 0.034210862 & 0.001170383 \\
\hline Friday & $13 / 05 / 2016$ & 4.83 & 1.08295964 & 0.079697702 & 0.006351724 \\
\hline Monday & $16 / 05 / 2016$ & 5.21 & 1.07867495 & 0.075733388 & 0.005735546 \\
\hline Tuesday & $17 / 05 / 2016$ & 5.05 & 0.96928983 & -0.03119161 & 0.000972917 \\
\hline Wed. & $18 / 05 / 2016$ & 5.1 & 1.00990099 & 0.009852296 & $9.70677 \mathrm{E}-05$ \\
\hline Thursday & $19 / 05 / 2016$ & 5.35 & 1.04901961 & 0.047856021 & 0.002290199 \\
\hline Friday & $20 / 05 / 2016$ & 5.35 & 1 & 0 & 0 \\
\hline Monday & $23 / 05 / 2016$ & 5.1 & 0.95327103 & -0.04785602 & 0.002290199 \\
\hline Tuesday & $24 / 05 / 2016$ & 5.2 & 1.01960784 & 0.019418086 & 0.000377062 \\
\hline Wed. & $25 / 05 / 2016$ & 5.61 & 1.07884615 & 0.075892094 & 0.00575961 \\
\hline Thursday & $26 / 05 / 2016$ & 5.98 & 1.06595365 & 0.063869848 & 0.004079358 \\
\hline Friday & $27 / 05 / 2016$ & 5.96 & 0.99665552 & -0.00335009 & $1.12231 \mathrm{E}-05$ \\
\hline Monday & $30 / 05 / 2016$ & 5.96 & 1 & 0 & 0 \\
\hline Tuesday & $31 / 05 / 2016$ & 5.67 & 0.95134228 & -0.04988136 & 0.00248815 \\
\hline Wed. & $01 / 06 / 2016$ & 5.28 & 0.93121693 & -0.07126302 & 0.005078418 \\
\hline Thursday & $02 / 06 / 2016$ & 5.29 & 1.00189394 & 0.001892148 & $3.58022 \mathrm{E}-06$ \\
\hline Friday & $03 / 06 / 2016$ & 5.56 & 1.0510397 & 0.049779862 & 0.002478035 \\
\hline Monday & $06 / 06 / 2016$ & 5.3 & 0.95323741 & -0.04789129 & 0.002293575 \\
\hline Tuesday & $07 / 06 / 2016$ & 5.3 & 1 & 0 & 0 \\
\hline Wed. & $08 / 06 / 2016$ & 5.35 & 1.00943396 & 0.00938974 & $8.81672 \mathrm{E}-05$ \\
\hline Thursday & $09 / 06 / 2016$ & 5.5 & 1.02803738 & 0.027651531 & 0.000764607 \\
\hline Friday & $10 / 06 / 2016$ & 5.52 & 1.00363636 & 0.003629768 & $1.31752 \mathrm{E}-05$ \\
\hline Monday & $13 / 06 / 2016$ & 5.75 & 1.04166667 & 0.040821995 & 0.001666435 \\
\hline Tuesday & $14 / 06 / 2016$ & 5.79 & 1.00695652 & 0.006932437 & $4.80587 \mathrm{E}-05$ \\
\hline Wed. & $15 / 06 / 2016$ & 6 & 1.03626943 & 0.035627178 & 0.001269296 \\
\hline Thursday & $16 / 06 / 2016$ & 5.99 & 0.99833333 & -0.00166806 & $2.78241 \mathrm{E}-06$ \\
\hline Friday & $17 / 06 / 2016$ & 5.92 & 0.98831386 & -0.01175496 & 0.000138179 \\
\hline Monday & $20 / 06 / 2016$ & 5.8 & 0.97972973 & -0.02047853 & 0.00041937 \\
\hline Tuesday & $21 / 06 / 2016$ & 6.1 & 1.05172414 & 0.050430854 & 0.002543271 \\
\hline Wed. & $22 / 06 / 2016$ & 6.2 & 1.01639344 & 0.016260521 & 0.000264405 \\
\hline Thursday & $23 / 06 / 2016$ & 6.16 & 0.99354839 & -0.00647251 & $4.18934 \mathrm{E}-05$ \\
\hline Friday & $24 / 06 / 2016$ & 5.93 & 0.96266234 & -0.03805256 & 0.001447998 \\
\hline Monday & $27 / 06 / 2016$ & 5.9 & 0.99494098 & -0.00507186 & $2.57238 \mathrm{E}-05$ \\
\hline Tuesday & $28 / 06 / 2016$ & 5.8 & 0.98305085 & -0.01709443 & 0.00029222 \\
\hline Wed. & $29 / 06 / 2016$ & 5.71 & 0.98448276 & -0.01563889 & 0.000244575 \\
\hline Thursday & $30 / 06 / 2016$ & 5.71 & 1 & 0 & 0 \\
\hline Friday & $01 / 07 / 2016$ & 5.75 & 1.00700525 & 0.006980831 & $4.8732 \mathrm{E}-05$ \\
\hline Sum & & & & 0.295246845 & 0.050823065 \\
\hline
\end{tabular}


The mean daily return

$$
\bar{\mu}=\frac{0.295246845}{40}=0.007381171125
$$

Standard deviation of daily returns from the sample of access stock is:

$$
\sqrt{\frac{0.050823065}{39}-\frac{(0.295246845)^{2}}{40(39)}}=\sqrt{0.0012472773}=0.0353168 \text {. }
$$

We annualize this estimate of the standard deviation by assuming that there are about 252 trading days in a year, and obtain

$$
\hat{\sigma}=\left\{\frac{s}{\sqrt{\tau}}\right\}=\left\{\frac{0.0353168}{\sqrt{\frac{1}{252}}}\right\}=0.0353168 \times \sqrt{252}=0.560636818
$$

Thus, the estimated annualized volatility measure (standard deviation of Bank $\mathrm{X}$ stock is 0.560. Therefore, in the Black-Scholes formulation, Bank X returns is assumed to follow a normal distribution with the estimated mean $\mu=0.0073812$ as the drift, and standard deviation $\sigma=0.5606368$ which measures the volatility. The following code uses these statistics to closely approximate Bank X's stock price dynamics using the EM method.

Figure 5 shows, as earlier stated, that Euler-Maruyama approximation is a close estimate to solutions of stochastic differential equation of interest, thus making it possible for us to adopt the same approach especially when the analytical solutions are not feasible or difficult to obtain for any SDEs of interest.

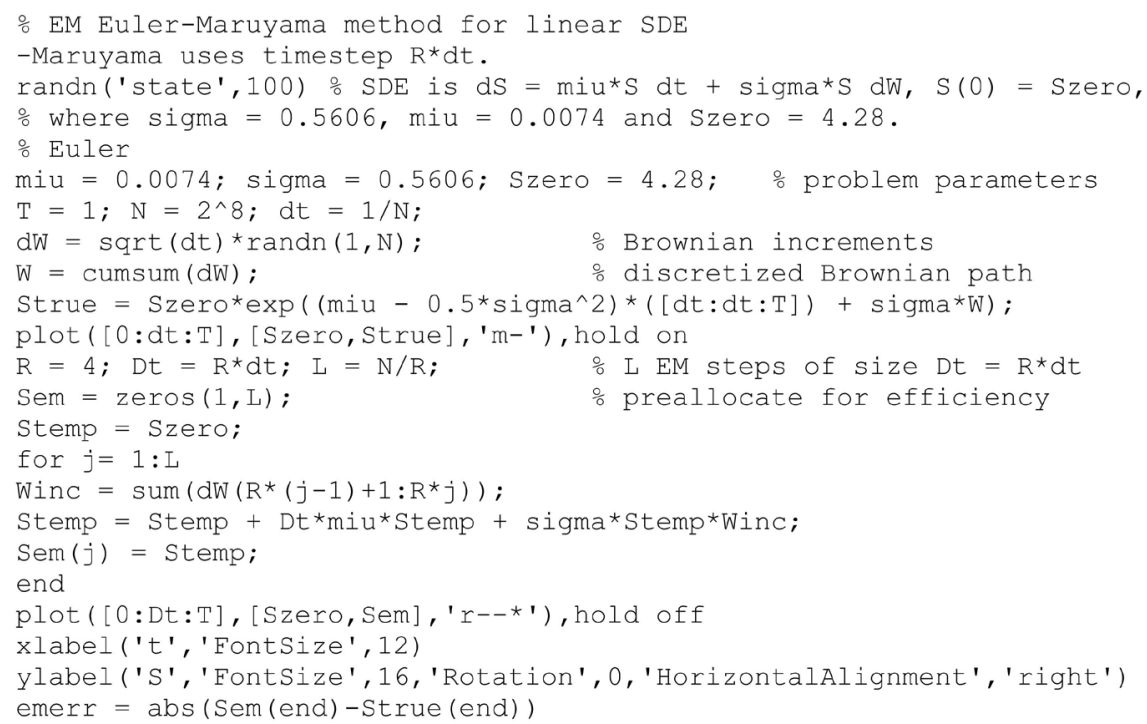

The error between the Euler-Maruyama and exact solution represented by emerr is given by

$$
\text { emerr }=\operatorname{abs}(\operatorname{sem}(\text { end })-\text { Strue }(\text { end }))=0.2413
$$

which could be minimized by an appropriate choice of $\mathrm{R}$ as stated before. 


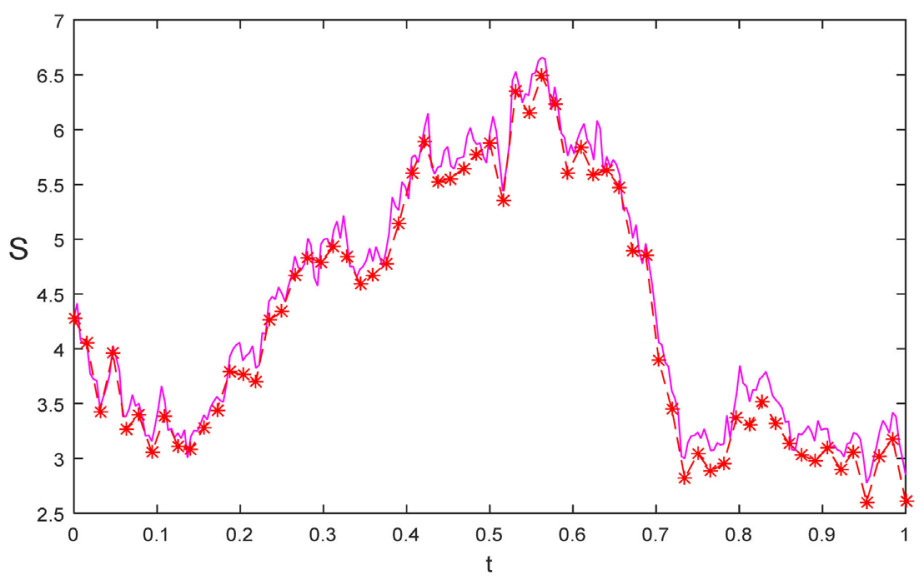

Figure 5. Exact and EM approximations for bank X prices.

From Equations (3)-(5) the Black-Scholes formula for a European call option pricing is given by:

\[ (S, T)=S N\left(d_{1}\right)-K \exp (-r T) N\left(d_{2}\right) \]
where $d_{1}=\frac{\ln \left(\frac{S}{K}\right)+\left(r+\frac{\sigma^{2}}{2}\right) T}{\sigma \sqrt{T}}$ and $d_{2}=d_{1}-\sigma \sqrt{T}=\frac{\ln \left(\frac{S}{K}\right)+\left(r-\frac{\sigma^{2}}{2}\right) T}{\sigma \sqrt{T}}$.

For the put option in the European type of option pricing we have:

$$
P(T)=\operatorname{Kexp}(-r T) N\left(d_{2}\right)-S N\left(d_{1}\right)
$$

where $d_{1}=\frac{\ln \left(\frac{S}{K}\right)+\left(r+\frac{\sigma^{2}}{2}\right) T}{\sigma \sqrt{T}}$ and $d_{2}=d_{1}-\sigma \sqrt{T}=\frac{\ln \left(\frac{S}{K}\right)+\left(r-\frac{\sigma^{2}}{2}\right) T}{\sigma \sqrt{T}}$.

Or in a more general and compact form we could write it as:

$$
\begin{gathered}
C(S, t)=S N\left(d_{1}\right)-K \exp (-r \tau) N\left(d_{2}\right) \\
\text { and } P(S, t)=K \exp (-r \tau) N\left(d_{2}\right)-S N\left(d_{1}\right) \\
d_{1}=\frac{\ln \left(\frac{S}{K}\right)+\left(r+\frac{\sigma^{2}}{2}\right) \tau}{\sigma \sqrt{\tau}} \text { and } d_{2}=d_{1}-\sigma \sqrt{\tau}=\frac{\ln \left(\frac{S}{K}\right)+\left(r-\frac{\sigma^{2}}{2}\right) \tau}{\sigma \sqrt{\tau}}
\end{gathered}
$$

$C(S, t)=$ Price of the European call option, $P(S, t)=$ Price of the European put option, $S=$ Current underlying asset (stock) price, $K=$ Strike price, $\tau=T-t$, is the current annualized time-to-expiration, where $T$ is the expiration date, $r=$ The annualized risk-free interest rate, $\sigma=$ The annualized standard deviation of the underlying asset price, and $N=$ The cumulative distributions function for a standard normal variable.

We present below the MATLAB code for the computation of a European put-option using the Black-Scholes Formula:

\subsection{Langevin Equation (Ornstein-Uhlenbeck Process)}

This equation models mean-reverting processes such as interest rates. This sec- 
tion is important for future work in interest rate derivatives in the NSM, given the fundamental role played by interest rates in financial and national economic management. Consider an SDE of the form:

$$
\mathrm{d} X(t)=-\mu X(t) \mathrm{d} t+\sigma \mathrm{d} W(t)
$$

where $\mu$ and $\sigma \in \mathbb{R}^{+}$. The solution to this type of equation is called Ornstein-Uhlenbeck process. The Euler-Maruyama and Milstein's approximation methods are identical here, since there are no $X(t)$ term in the diffusion component. It is difficult to obtain an analytic solution to Equation (18) above, as was the case with the geometric Brownian motion seen earlier. To confirm this, we solve the SDE (18) and examine the nature of the solution. We observe that $\mathrm{d} X(t)+\mu X(t) \mathrm{d} t=\sigma \mathrm{d} W(t)$ so that multiplying both sides by the integrating factor we shall obtain:

$$
\mathrm{d}\left(\mathrm{e}^{\mu t} X(T)\right)=\mu X(t) \mathrm{e}^{\mu t} \mathrm{~d} t+\mathrm{e}^{\mu t} \mathrm{~d} X(t)=\mathrm{e}^{\mu t} \sigma \mathrm{d} W(t)
$$

Integrating both sides gives;

$$
\begin{gathered}
\left.\mathrm{e}^{\mu t} X(t)\right|_{0} ^{t}=\sigma \int_{0}^{t} \mathrm{e}^{\mu s} \mathrm{~d} W(s) \\
\Rightarrow \mathrm{e}^{\mu t} X(t)-\mathrm{e}^{0} X(0)=\sigma \int_{0}^{t} \mathrm{e}^{\mu s} \mathrm{~d} W(s) \\
\Rightarrow \mathrm{e}^{\mu t} X(t)-X(0)=\sigma \int_{0}^{t} \mathrm{e}^{\mu s} \mathrm{~d} W(s) \\
\Rightarrow X(t)=X(0) \mathrm{e}^{-\mu t}+\sigma \int_{0}^{t} \mathrm{e}^{\mu(s-t)} \mathrm{d} W(s)
\end{gathered}
$$

The second term on the right-hand side shows that no closed form solution exists and that the only solution is the non-trivial one. Hence, we need a numerical simulation to the SDE, unlike the Brownian motion where we have the trivial (closed form) solution as well as the numerical approximations.

Figure 6 is a typical illustration of numerical solution of stochastic differential equation model where the analytical solution is difficult to achieve. Such models as interest rate are evaluated through this type of numerical approximation. Interest rate dynamics as one of the factors that determine prices which are of interest to investors and policy makers in the Nigerian Market could be approximated numerically using this model. This will provide good estimates in the short run to the movement of interest rate in any given market, NSM inclusive.

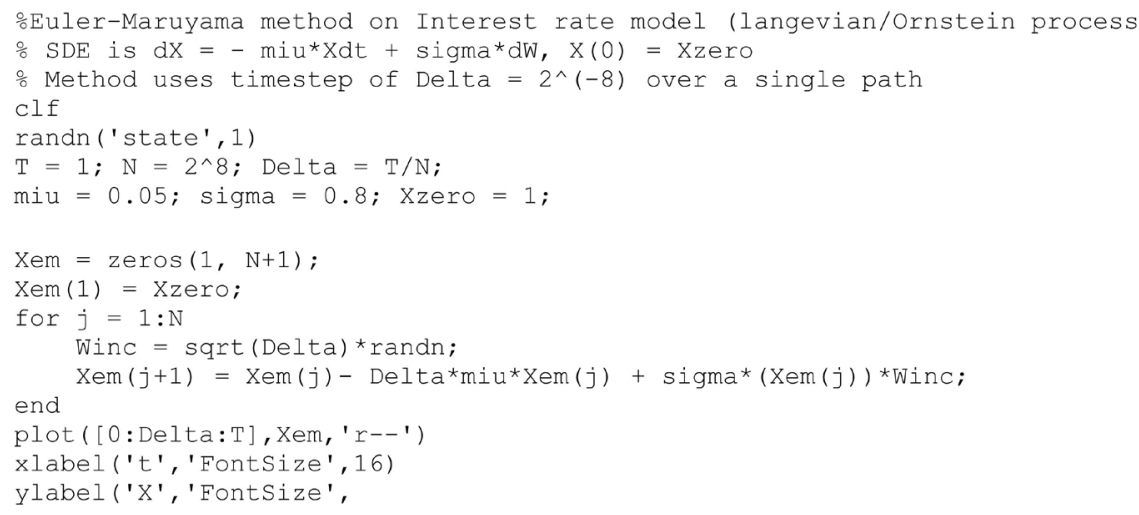




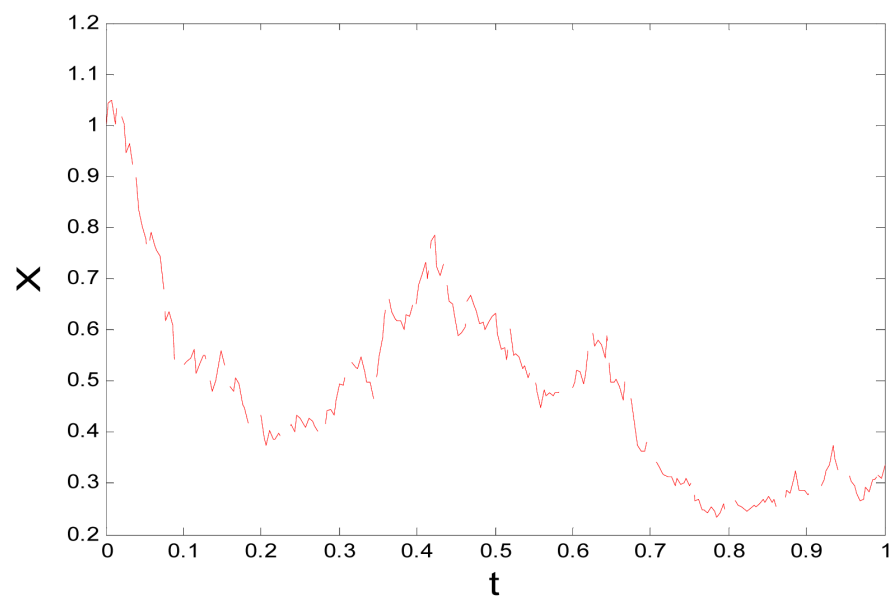

Figure 6. EM approximation for European interest rate put option.

\subsection{Errors in Euler-Maruyama and Milstein's Approximation}

Usually, analytical solutions of many SDEs are not known explicitly and therefore require simulations. However, when the explicit solution to a given SDE is known, it is realistic to obtain the absolute error of approximation, defined by [33] as the expectation of the absolute value of the difference between the approximation and the Ito process at time $T$, and given by

$$
\varepsilon_{\Delta}=E\left(\left|X_{\text {app }}\left(t_{i}\right)-X_{\text {true }}\left(t_{i}\right)\right|\right)
$$

where $t_{i}=i \Delta ; i=1,2, \cdots, N$ and $E$ denotes the mean value. By computing $N$ different simulations of sample paths of the Ito Calculus process and their respective Euler-Maruyama approximations corresponding to the same sample paths of the Wiener process, we estimate the absolute error $\varepsilon$ by

$$
\widehat{\varepsilon_{\Delta}}=\frac{1}{N} \sum_{i=1}^{N}\left|X_{\text {app }}\left(t_{i}\right)-X_{\text {true }}\left(t_{i}\right)\right|
$$

Convergence: Let $X_{n}$ and $X\left(t_{n}\right)$ be random variables for all $t_{n}$. Strong and weak convergence concepts are important here. Weak convergence refers to error of the mean and strong convergence is concerned with mean of the error. [39] defines weak convergence as follows. Given a function $\Phi$, the weak error is

$$
\varepsilon_{\Delta t}^{\text {weak }}:=\sup _{0 \leq t_{n} \leq T}\left|E\left[\Phi\left(X_{n}\right)\right]-E\left[\Phi\left(X\left(t_{n}\right)\right)\right]\right| \leq C \Delta t^{\gamma}
$$

In other words, $\varepsilon_{\Delta t}^{\text {weak }} \rightarrow 0$ as $\Delta t \rightarrow 0$. The expression $E\left[\Phi\left(X_{n}\right)\right]$ can be estimated by Monte Carlo simulation over many paths. For strong convergence (which is the mean of the error), we have

$$
\varepsilon_{\Delta t}^{\text {strong }}:=\sup _{0 \leq t_{n} \leq T} E\left[\left|X_{n}-X\left(t_{n}\right)\right|\right] \leq C \Delta t^{\gamma}
$$

Hence, the random variable converges strongly if $\varepsilon_{\Delta t}^{\text {strong }} \rightarrow 0$ as $\Delta t \rightarrow 0$. Thus, we can always estimate the errors in our numerical approximation to determine whether there is strong or weak convergence using the least square fit. In doing this we note that the Euler-Maruyama numerical approximation has a weak order of convergence $(\gamma=1)$ and a strong order of convergence $\left(\gamma=\frac{1}{2}\right)$. 
This could be estimated using the relation

$$
\varepsilon(\Delta t)=C \cdot(\Delta t)^{\gamma} \Leftrightarrow \log [\varepsilon(\Delta t)]=\log C+\gamma \log (\Delta t)
$$

These error equations will be used in the experimental modelling of derivative products in the NSM to check for fast convergence of the pricing models under different scenarios (parameter values) and underlying assumptions, and heuristics of JSE-NSM price dynamics. This follow-on work was mentioned in the introduction to this paper. To further explain the contributions of this paper to current and future research on introducing derivative pricing in the NSM, we summarise below the heuristics behind using existing JSE derivatives to explore suitable and currently non-existent NSM ones. The paper principally justifies the choice of suitable $\mathrm{R}$ values above and the nice convergence properties of the EM schema in the heuristics.

\subsection{Policy Implications and Heuristics for Modelling JSE-NSM Derivative Price Dynamics}

The management of the Nigerian Stock Exchange (NSE) indicated in a meeting with the researchers in 2014 that the NSM was interested in using derivative products to: deepen the markets; enable such products to play traditional roles in risk hedging, speculation and arbitrage; and successfully benchmark its performance on existing JSE derivatives, given the relatively more advanced status of the latter. Hence, the heuristics aims to combine JSE derivatives data with broader NSM stylized facts and characterisations, especially based on Random Matrix Theory (RMT), to simulate plausible derivative models and prices that will fit the Nigerian stylized facts and RMT results better. For example, to cover the essential scope in this initial modelling of derivatives in the NSM, we will look at key sectors and products that will be more useful for achieving the stated derivative modelling objectives-risk hedging, speculation and arbitrage-especially those which the NSE management mentioned that major NSM investors are interested in.

As mentioned in the introduction to this paper, these products include currency options, cross-currency swaps, deliverable and non-deliverable forwards. Also, important market sectors in these considerations are banking and financial services (as in this paper), energy and (agricultural) commodity derivatives such as futures, because of the strategic relevance of energy and agriculture sectors in the Nigerian economy. For instance, banking and financial services are fundamental sources of development finance for investors (households, firms and government).Oil and gas provide the energy inputs into manufacturing and production of goods and services, and revenues for Nigeria, and agricultural products support other industries.

The ingredients for the derivative pricing heuristics are the correlation structures from RMT analyses and Black-Scholes derivative pricing models, observed stylized facts of underlying asset prices, and implied volatility dynamics in the JSE. These facts will be categorised as Generalised Stylised Facts (GSFs) and Im- 
plied Volatility Stylised Facts (IVSFs). Sequential modelling in form of models M1, M2, M3, for example, will exploit the JSE data, based on comparative analysis of the performance of selected derivative models against the standard BS model, for suitable derivative products mentioned above. The reason for this approach is to understand which BS models or extensions of the BS model are typically used in the JSE for specific asset prices, and whether the derivative prices from competing models are more accurate than the ones used.

This knowledge will be very useful to NSE management, as they optimise the decision choices facing them in introducing derivative products and models in the NSM. It will also be useful to the JSE management, if they become aware that existing models used in pricing JSE derivatives are not as good as alternative models revealed by this research. This, therefore, will be a crucial contribution of the heuristics to knowledge.

We, however, recall that the underlying asset prices are available in Nigeria, but not derivative prices. Given that derivative models are common theoretical knowledge across the two markets, we represent the Nigerian information as NBS for BS model, NGSFs for General Stylised Facts (GSFs), NUAPs for underlying asset prices. We use the known NGSFs in Nigeria to estimate the unknown Nigerian implied volatility stylised facts (NIVSFs). Similar notations are adopted for JSE by replacing by $\mathrm{S}$. The key research question to explore in a follow-on work to this paper is: How do we overcome the lack of research data on the IVSFs which underpin derivative pricing in the NSM?

In brief, the following steps are involved: 1) compare the generalised stylised facts (GSF information) on underlying prices for JSM and NSM, to gauge how close the two data sets are in behaviour (using, say, the first four moments and distributions of the data sets); 2) explore the correlation or heuristic links between the full data on SGSFs and SIVSFs in South Africa, and across Nigeria and South Africa; 3) infer therefore the likely range of values for the unknown Nigerian IVSFs; 4) run RMT analyses on asset prices from key market sectors in NSM and JSM (for example selected banks, oil and gas, commodities), to characterise mainstream tendencies in the markets, and further refine the initial correlational and heuristic links in b) above; e) using knowledge of the RMT comparisons, simulate plausible data that fits the Nigerian modelling scenarios and repeat the sequence of modelling M1, M2, M3, .., on the data, [40]. This will produce indicative results which will inform possible decisions on the models and projected prices that could obtain in the NSM, under different modelling scenarios and assumptions.

\subsection{Further Description of the Heuristics}

This section explains the strategy for modelling the currently non-existing data on derivative pricing in Nigeria. Heuristics generally refers to the use of creative common-sense reasoning to perform tasks that ordinarily would have been (near) impossible to do. This impossibility trait explains why there is no known result on derivative trading and pricing in Nigeria because qualified financial 
engineers argue that there is no historical data to work with. We reiterate that successful application of this strategy in future work will constitute a novel methodological, theoretical and practical contribution of the research to knowledge.

As noted above, the NSM is benchmarking its performance on the Johannesburg Stock Exchange (JSE), given the relatively more advanced status of the JSE. We know that the JSE has been trading on different types of derivatives. Hence, the purpose of this statement of strategy is to explain how existing knowledge of derivatives in the JSE will be combined with analysis of broader stock market features (stylized facts) and characterisations, especially based on Random Matrix Theory (RMT), to simulate plausible derivative models and prices that will fit the Nigerian data (stylized facts and RMT results) better. The following schema in Table 1 explains visually the above-mentioned steps involved in this strategy.

The figure is presented as a quadrant with one half representing the Nigerian side of the intended analysis, and the other side the South Africa side. Information from the South African side will underpin the specific (simulated data modelling in Nigeria). Column 1 of the figure summarises the nature of products of interest. For example, to cover the essential scope in this initial modelling of derivatives in the NSM the researcher could look at key sectors and products that will be more useful for achieving the stated derivative modelling objectives-risk hedging, speculation and arbitrage-especially those that the NSE management mentioned that major NSM investors are interested in. These products include currency options, cross-currency swaps, deliverable and non-deliverable forwards. Also, important in these considerations are energy and (agricultural) commodity derivatives such as futures, because of the strategic relevance of energy and agriculture sectors in the Nigerian economy. For instance, oil and gas in addition to providing the energy inputs into manufacturing and production of

Table 1. A visual schematic for comparative modelling of derivative in JSM and NSM.

\begin{tabular}{|c|c|c|}
\hline Products sectors/models & Information in Johannesburg Stock Market (JSM) & Information in Nigerian Stock Market (NSM) \\
\hline $\begin{array}{c}\text { Currency options, } \\
\text { Cross-currency swaps, } \\
\text { Forwards }\end{array}$ & $\begin{array}{l}\text { South African Asset Prices, BS Derivative prices, } \\
\text { South African Generalised Stylised Facts (GSFs) }\end{array}$ & $\begin{array}{l}\text { Nigerian Underlying Asset Prices (NUAPs), } \\
\text { (NIVSFs), Nigerian Generalise Stylised Facts (NGSFs) }\end{array}$ \\
\hline $\begin{array}{l}\text { Financial Services, Energy } \\
\text { and Agricultural sectors }\end{array}$ & $\begin{array}{l}\text { South African Implied Volatility } \\
\text { Stylised Facts (IVSFs) (All known) }\end{array}$ & $\begin{array}{l}\text { Nigerian Implied Volatility Stylised Facts } \\
\text { (IVSFs)-To be simulated }\end{array}$ \\
\hline Models & Steps in the modelling & Steps in the modelling \\
\hline $\begin{array}{c}\text { Black-Scholes, } \\
\text { Ad-Hoc Black-Scholes } \\
\text { and other derivative } \\
\text { pricing models }\end{array}$ & $\begin{array}{l}\text { 1) Test Black-Scholes alternative derivative } \\
\text { pricing models on some South African data } \\
\text { 2) Use known model assumptions and data behaviour } \\
\text { (stylized facts) to obtain } M_{1}, M_{2}, M_{3} \text { models } \\
\text { 3) Run suitable RMT analysis } \\
\text { 4) If possible ascertain underpinning } \\
\text { data distributions } \\
\text { 5) Determine optimal models from } M_{1}, M_{2}, M_{3}\end{array}$ & $\begin{array}{l}\text { 1) Repeat Random matrix theory analysis } \\
\text { on similar NSM data as in JSM } \\
\text { 2) Fit suitable distribution to NSM data (Generalized } \\
\text { distribution, (GD and Generalized stylized facts, GS) } \\
\text { 3) Compare } 1 \text { and } 2 \text { with JSM results and simulate } \\
\text { likely Implied volatility surfaces (IVSFs) } \\
\text { 4) Use insights from } 1 \text { - } 3 \text { above to simulate } \\
\text { corresponding NSM data and } M_{1}, M_{2}, M_{3} \\
\text { models from Nigeria. }\end{array}$ \\
\hline
\end{tabular}


goods and services are key revenue earners for Nigeria and agricultural products support other industries. Another key sector of potential interest in this work, especially in connection with RMT characterisation is the banking sector, again because of the overarching importance of banks source of development finance for investors (households, firms and government). The researcher will explain in simple terms the role of RMT in the research shortly.

Column 2 depicts the nature of empirical modelling to be performed on existing South African data. The upper-left quadrant of the column uses the symbolisms $\mathrm{B}, \mathrm{S}$, and $\mathrm{D}$ to portray indicative analyses using the Black-Scholes derivative pricing model (B) on observed stylized facts (S) of data on underlying asset prices and implied volatility dynamics (D) in the JSE. These data are represented as Generalised Stylised Facts (GSFs) and Implied Volatility Stylised Facts (IVSFs). The lower-left quadrant depicts the nature of sequential modelling $M_{1}$, $M_{2}, M_{3}, \ldots$ which will exploit the JSE data, based on comparative analysis of the performance of selected derivative models (see Chapter 2 on literature review of the various models) against the standard BS model, for suitable derivative products mentioned above, as appropriate.

Column 3 depicts the nature of empirical modelling to be performed on the as yet unavailable Nigerian implied volatility data. Recall that the underlying asset prices are available in Nigeria, but not derivative prices. Given that derivative models are common theoretical knowledge across the two markets, we represent the Nigerian information as B for BS model, GS for General Stylised Facts (GSFs), and so on.

The above-mentioned steps in modelling derivatives in Nigeria through revealed affinities between the NSM and JSM trading data will be achieved within a broad-based characterisation work using suitable systems of sector- and asset-based stylised facts and RMT results. RMT is a theoretical suite of techniques which originated from statistical physics and econophysics. It primarily uses correlation measures among clusters of measurements, based on eigenvalue and eigenvector analyses, among other techniques in multivariate statistics, underpinned by assumptions about the likely types of probability distributions which generate the data clusters, to explore the relationships among the data clusters.

Hence, RMT techniques will be used as baseline tools for initially studying the closeness or otherwise among the selected data clusters from sectors and sets of asset prices in the JSM and NSM. The results will then be combined with further knowledge of 1) the statistical distributions which govern the respective data cluster, 2) the extent to which the data behaviours support the assumptions of different derivative pricing models, with the BS model as a reference point, hence the plausible validity of the models in deciphering derivative prices, in order to simulate supposed NSM data that fit the distributions and models, and thereby produce plausible derivative prices for the NSM. Importantly, the results will provide theoretical knowledge of the limitations of different derivative pricing models. 


\section{Summary and Conclusions}

This paper showed that the differences between analytical solutions to derivatives pricing SDEs and their Euler-Maruyama numerical approximations, which depend on the values of the drift and volatility parameters of derivative pricing models, and the initial take off price of the asset denoted by $S_{0}$, are meaning fully negligible, generally and for the Bank X data, to warrant further use of the approximations in derivative pricing in the NSM.

There were remarkable differences between the exact or analytical values and that of Euler-Maruyama (EM) approximations for sufficiently large value of $\mathrm{R}$. For smaller values of the parameters, the plotted analytical (exact) solution coincided with that of Euler-Maruyama approximation. From the Bank X data, therefore, the EM solution is essentially the same with the true solution, even for large values of $\mathrm{R}(\Delta t)$. In other words, the EM approximation could be used to estimate financial asset prices such as equity derivatives in the Black-Scholes model and interest rate derivatives in the Ornstein Uhlenbeck model, which in turn is needed in derivative asset pricing, re the rates of return in the models. The procedure can also be extended to constant elasticity of variance (CEV) model given by

$$
\mathrm{d} S=\mu S \mathrm{~d} t+\sigma S^{\frac{\alpha}{2}} \mathrm{~d} W
$$

where the model was estimated using EM approximation method as shown (for $\alpha=1$, results suppressed). Market participants can thus use these properties of EM approximations in forecasting the values of assets in their portfolio of investment, for appropriate pricing of such securities in derivative contracts.

The results give some confidence that these standard approaches to asset price simulation are applicable to the NSM setting, a fact that provides a background for more intense computational modelling of derivative prices in the NSM, under different model assumptions, and for different ranges of the drift and initial price parameters applicable in different sectors of the NSM, with good potential for supporting derivatives trading. We noted earlier in the introduction to this paper that this larger problem of successfully preparing the grounds for derivatives trading in the NSM motivates the paper, and is typically approached by combining ideas from the heuristics of JSE-NSM derivatives and asset prices, Monte-Carlo simulation, and Ito Calculus. We expatiated on these points in the foregoing notes on heuristic modelling of NSM derivative prices using known information from JSE.

This is the first time that such a line of work will be conducted in the NSM, well before such derivatives are fully introduced. Future work consists in finding through the EM approximation schema optimal derivative pricing models which will be suitable for the NSM. The experimental modelling of prices foreshadowed above requires testing out a spectrum of candidate pricing models, with different assumptions, for different products and sectors of the NSM. This is the sense in which this paper and related future work so described are part of 
SSMCD work by the authors.

Further implications of this line of work are such that academics and practitioners can use the results as starting points for enhancing the research and practice of derivative pricing in the NSM and other emerging markets, for sectors and products of interest to them. The novelty of this line of work is that it has not been done so far in the NSM, and wider emerging African markets. Hence, it has immense potential for systematically characterising such markets. Moreover, using the results to deepen the markets via derivative products is the overriding policy goal of this and related papers.

Indeed, this paper intends to help the NSE management to use derivatives to deepen the NSM. The specific objectives of the paper and the above notes on policy implications provide the rudiments of theory and follow-on heuristics for this goal. Immediate future work is on applying the outlined heuristics to develop the pioneer derivative products that will be suitable for the NSM. This will require more detailed work on the stylistics of key variables associated with NSM asset prices and returns.

\section{Acknowledgements}

We are grateful for the sponsorship of the $\mathrm{PhD}$ research of which this paper is a part by the Nigerian Tertiary Education Fund.

\section{Conflicts of Interest}

The authors declare no conflicts of interest regarding the publication of this paper.

\section{References}

[1] Black, F. and Scholes, M. (1973) The Pricing of Options and Liabilities. The Journal of Political Economy, 81, 637-654. https://doi.org/10.1086/260062

[2] John, C.H. (2000) Options, Futures and Other Derivatives. Prentice Hall, London.

[3] African Development Bank Group (2010) African Fixed Income and Derivative Guidebook.

[4] Ezepue, P.O. and Omar, M.A.T. (2012) Weak-Form Market Efficiency of the Nigerian Stock Market in the Context of Financial Reforms and Global Financial Crisis. Journal of African Business, 13, 209-220. https://doi.org/10.1080/15228916.2012.727750

[5] Omar, M.A.T. (2012) Stochastic Models in Emerging Financial Markets: A Case Study of the Nigerian Stock Market. Ph.D. Dissertation, Sheffield Hallam University, UK.

[6] Mullins, M. and Murphy, F. (2009) Financial Globalization, State Economy and Modern Financial Instruments: The Case of Brazil. Globalizational Journal, 6, 433-499. https://doi.org/10.1080/14747730903298645

[7] Aysun, U. and Guldi, M. (2011) Derivatives Market Activity in Emerging Markets and Exchange Rate Exposure. Journal of Emerging Markets Finance and Trade, 47, 46-67. https://doi.org/10.2753/REE1540-496X470603

[8] Ameer, R. (2009) Value-Reliance of Foreign Exchange and Interest Rate de Discourse: The Case of Malaysia Firms. Journal of Risk Finance, 10, 78-90. 
https://doi.org/10.1108/15265940910924517

[9] Dodd, R. and Griffith-Jones, S. (2007) Report on Derivative Markets: Stabilizing or Speculative Impact on Chile and a Comparison with Brazil. A Project Document of United Nations in Economic Commission for Latin America and the Caribbean (ECLAC).

[10] Ezepue, P.O. and Raheem, M.A. (2016) CBN Bank Reforms, Global Financial Crisis and Volatilities of Nigerian Bank Returns. Proceedings of the International Symposium on Mathematical and Statistical Finance, 1-3 September 2015, University of Ibadan, Ibadan.

[11] Panzar, L. and Cipu, E.C. (2004) Using of Stochastic Ito and Stratonovich Integrals in Derived Security Pricing. Proceedings of the 3rd International Colloquium "Mathematics in Engineering and Numerical Physics", Bucharest, 7-9 October 2004, 222-229.

[12] Raheem, M. and Ezepue, P. (2018) Some Stylized Facts of Short-Term Stock Prices of Selected Nigerian Banks. Open Journal of Statistics. https://doi.org/10.4236/ojs.2018.81008

[13] Malliaris, A.G. and Brook, W. (1982) Stochastic Methods in Economics and Finance: Advanced Textbooks in Economics. North Holland Publishers, Elsevier and Amsterdam.

[14] Constantinides, G.M. (1978) Market Risk Adjustment in Project Valuation. The Journal of Finance, 33, 603-616. https://doi.org/10.1111/j.1540-6261.1978.tb04870.x

[15] Maruyama, G. (1955) Continuous Markov Processes and Stochastic Equations, Rendiconti del Circolo Matematico di Palermo, 4, 48-90. https://doi.org/10.1111/j.1540-6261.1978.tb04870.x

[16] McShane, E.J. (1974) Stochastic Calculus and Stochastic Models. Academic Press, New York.

[17] Milstein, G.N. (1974) Approximate Integration of Stochastic Differential Equations. Theory of Probability Applications, 19, 557-562.

[18] Kloeden, P.E. and Platen, E. (1989) A Survey of Numerical Methods for Stochastic Differential Equations. Stochastic Hydrology \& Hydraulics, 3, 155-178. https://doi.org/10.1007/BF01543857

[19] Newton, N.J. (1991) Asymptotically Efficient Runge-Kutta Methods for a Class of Ito and Stratonovich Equations. SIAM Journal of Applied Mathematics, 52, 542-567. https://doi.org/10.1137/0151028

[20] Saito, Y. and Mitsui, T. (1992) Discrete Approximation for Stochastic Differential Equations. Transactions of the Japan Society for Industrial and Applied Mathematics, 2, 1-16.

[21] Kloeden, P.E., Platen, E. and Scurz, H. (1993) Numerical Solution of Stochastic Differential Equations through Computer Experiments. Springer, Berlin.

[22] Ozaki, T. (1985) Nonlinear Time-Series Models and Dynamical Systems. In: Hannan, E.J., Krishnaiah, P.R. and Rao, M.M., Eds., Handbook of Statistics, North Holland, Amsterdam, 5.

[23] Ozaki, T. (1992) A Bridge between Nonlinear Time Series Models and Nonlinear Stochastic Dynamical Systems: A Local Linearization Approach. Statistica Sinica, 2, 113-135.

[24] Sauer, T. (2008) Finance Conference Proceedings. International Conference of Management, Developed and Hosted by the College of Information Sciences and Technology, The Pennsylvania State University.

[25] Richardson, M. (2009) Stochastic Differential Equations, Case Study. A Working 
Paper for Mathematical Modelling and Scientific Computing Group, Mathematical Institute, University of Oxford, 1-25.

[26] Desmond, J.H. (2001) An Algorithmic Introduction to Numerical Simulation of Stochastic Differential Equations. SIAM Review Journal, 43, 525-546.

[27] Sauer, T. (2013) Computational Solution of Stochastic Differential Equations. Advanced Review Periodicals. Computational Statistics, 5, 362-371. https://doi.org/10.1002/wics.1272

[28] Merton Robert, C. (1973) Theory of Rational Option Pricing. The Bell Journal of Economics and Management Science, 4, 141-183. https://doi.org/10.2307/3003143

[29] Brewer, K.D., Feng, Y. and Kwan, C.C.Y. (2012) Geometric Brownian Motion, Option Pricing and Simulation: Some Spreadsheet Based Exercises in Financial Modelling. e-Journal Spreadsheets in Education, 5, Article 4.

[30] Malliaris, A.G. and Brock, W.A. (1999) Stochastic Methods in Economics and Finance: Advanced Textbooks in Economics. North Holland Publishers, Elsevier, Amsterdam.

[31] Kunta, H. (1997) Stochastic Flows and Stochastic Differential Equations. Cambridge University Press, Cambridge.

[32] Gardiner, C.W. (1997) Handbook of Stochastic Methods for Physics, Chemistry and the Natural Sciences. Second Edition, Springer, Berlin.

[33] Baazaoui, R. and Ouerdiane, H. (2014) A Comparative Study of Numerical Simulation of Stochastic Differential Equations; Application to Stochastic Duffing-Oscillator. International Journal of Mathematical Analysis, 8, 229-245.

[34] Dunbar Steven, R. (2014) Stochastic Processes and Advanced Mathematical Finance: Stochastic Differential Equations and the Euler-Maruyama Method. A Working Paper, Department of Mathematics, University of Nebraska-Lincoln, Lincoln. http://www.math.unl.edu/sdunbarl

[35] Maruf, A.R. and Ezepue, P.O. (2016) A Three State Markov Model for Predicting Movements of Asset Returns of a Nigerian Bank. CBN Journal of Applied Statistics, 7, 77-99.

[36] Nnanwa, C.P., Urama, T.C. and Ezepue, P.O. (2017) Random Matrix Theory Analysis of Cross-Correlation in the Nigerian Stock Exchange. Proceedings of the International Scientific Forum.

[37] Urama, T.C., Ezepue, P.O. and Nnanwa, P.C. (2017) Analysis of Cross-Correlations in Emerging Markets Using Random Matrix Theory. Journal of Mathematical Finance, 7, 291-301. http://www.scirp.org/journal/jmf https://doi.org/10.4236/jmf.2017.72015

[38] Urama, T.C., Ezepue, P.O. and Nnanwa, P.C. (2017) Application of Random Matrix Theory in Estimating Realistic Implied Correlation Matrix from Option Prices. Proceedings of 6 th Annual International Conference on Computational Mathematics, Computational Geometry \& Statistics, (CMCGS), 2017 and 5th Annual International Conference on Operations Research and Statistics (ORS) 2017, Singapore.

[39] Desmond, J.H. (2010) Euler-Maruyama Approximation to Stochastic Differential Equation. A Working Paper, Department of Mathematics and Statistics, University of Strathclyde (Science), Basel.

[40] Voss, J. (2014) An Introduction to Statistical Computing: A Simulation-Based Approach. Wiley, Hoboken. 